\title{
Conversion of $\alpha, \beta$-Unsaturated Aldehydes into Saturated Esters: An Umpolung Reaction Catalyzed by Nucleophilic Carbenes
}

\author{
Audrey Chan and Karl A. Scheidt* \\ Department of Chemistry, Northwestern University, 2145 Sheridan Road, Evanston, Illinois, \\ 60208
}

\section{Supporting Information}

General Information. All reactions were carried out under a nitrogen atmosphere in flamedried glassware with magnetic stirring. THF, $\mathrm{Et}_{2} \mathrm{O}, \mathrm{CH}_{2} \mathrm{Cl}_{2}$, DMF and toluene were purified by passage through a bed of activated alumina. ${ }^{1}$ Reagents were purified prior to use unless otherwise stated following the guidelines of Perrin and Armarego. ${ }^{2}$ Purification of reaction products was carried out by flash chromatography using EM Reagent silica gel 60 (230-400 mesh). Analytical thin layer chromatography was performed on EM Reagent $0.25 \mathrm{~mm}$ silica gel $60-\mathrm{F}$ plates. Visualization was accomplished with UV light and anisaldehyde, ceric ammonium nitrate stain, potassium permangenate, or phosphomolybic acid followed by heating. Infrared spectra were recorded on a Perkin Elmer 1600 series FT-IR spectrometer. ${ }^{1} \mathrm{H}-\mathrm{NMR}$ spectra were recorded on a Varian Inova $500(500 \mathrm{MHz})$ or Mercury $400(400 \mathrm{MHz})$ spectrometer and are reported in ppm using solvent as an internal standard $\left(\mathrm{CDCl}_{3}\right.$ at $\left.7.26 \mathrm{ppm}\right)$. Data are reported as (ap = apparent, $\mathrm{s}=$ singlet, $\mathrm{d}=$ doublet, $\mathrm{t}=$ triplet, $\mathrm{q}=$ quartet, $\mathrm{m}=$ multiplet, $\mathrm{b}=$ broad; coupling constant(s) in Hz; integration. Proton-decoupled ${ }^{13} \mathrm{C}-\mathrm{NMR}$ spectra were recorded on a Varian Inova $500(125 \mathrm{MHz})$ or Mercury $400(100 \mathrm{MHz})$ spectrometer and are reported in ppm using solvent as an internal standard $\left(\mathrm{CDCl}_{3}\right.$ at $\left.77.0 \mathrm{ppm}\right)$. Mass spectra data were obtained on a Varian 1200 Quadrupole Mass Spectrometer.

3-(4-chlorophenyl)cinnamaldehyde was prepared according to Kirsch. ${ }^{3}$ 4-Chlorocinnamaldehyde was prepared according to a procedure analogous to Moloney. ${ }^{4}$ 4-Methoxycinnamaldehyde was purchased from Acros Chemical Company and the remaining aldehydes were commercially available from Sigma-Aldrich Chemical Company and purified before use.

1. Pangborn, A. B.; Giardello, M. A.; Grubbs, R. H.; Rosen, R. K.; Timmers, F. J. Organometal. 1996, 15, 15181520.

2. Perrin, D. D. and Armarego, W. L. Purification of Laboratory Chemicals; 3rd Ed., Pergamon Press, Oxford. 1988.

3. (a) Hess, S.; Kirsch, G. Synthesis, 2001, 5, 755-758. (b) Kirsch, G.; Prim, D.; Leising, F.; Mignani, G. J. Heterocycl. Chem. 1994, 31, 1005-1009.

4. Baldwin, J.E.; Turner, S.C.M.; Moloney, M.G. Tetrahedron, 1994, 50, 9411-9424. 
Imidazolium salt $\mathbf{D}$ was prepared according to Diver and coworkers. ${ }^{5}$

General Procedure for benzimidazolium-catalyzed reaction:

A screw-capped tube was charged with the benzimidazolium salt $(11 \mathrm{mg}, 0.04 \mathrm{mmol})$ in a nitrogen-filled dry box. The tube was removed from the box and placed under a positive pressure of nitrogen. The tube was charged with toluene $(1.0 \mathrm{~mL}), \mathrm{DBU}(6 \mu \mathrm{L}, 0.04 \mathrm{mmol})$ and lastly, distilled cinnamaldehyde $(104 \mathrm{mg}, 0.79 \mathrm{mmol})$. The reaction mixture was allowed to stir for 5 minutes after which phenol $(150 \mathrm{mg}, 1.6 \mathrm{mmol})$ in toluene $(0.6 \mathrm{ml})$ was added via syringe followed by the addition of benzyl alcohol $(429 \mathrm{mg}, 4 \mathrm{mmol})$. The reaction was heated at 110 ${ }^{\circ} \mathrm{C}$ for 2-6 hours until cinnamaldehyde was consumed (as judged by GC). The reaction was cooled to room temperature, diluted with methylene chloride $(20 \mathrm{~mL})$ and washed with water $(20$ $\mathrm{mL})$. The aqueous layer was washed with methylene chloride $(3 \times 30 \mathrm{~mL})$ and the combined organic extracts were dried over anhydrous sodium sulfate, filtered, and concentrated in vacuo. The resulting residue was purified by flash column chromatography on silica gel.

Known compounds:

\begin{tabular}{|l|l|}
\hline & \multicolumn{1}{|c|}{ Reference } \\
\hline & $\begin{array}{l}\text { Rarton, P.; Laws, A.P.; Page, M.I. J. Chem. Soc. Perkin Trans. } 2 \\
\text { Rivero, I.A.; Heredia, S.; Ochoa, A. Synth. Commun. 2001, 31, } \\
2169-2176 .\end{array}$ \\
\hline & $\begin{array}{l}\text { Kurono, N.; Sugita, K.; Takasugi, S.; Tokuda, M. Tetrahedron, } \\
\mathbf{1 9 9 9 ,} 55,6097-6108 .\end{array}$ \\
\hline & $\begin{array}{l}\text { Kita, Y.; Akai, S.; Yamamoto, M.; Taniguchi, M.; Tamura, Y. } \\
\text { Synthesis, 1989, 4, 334-337. }\end{array}$ \\
\hline
\end{tabular}

\section{Selected Spectral Data:}

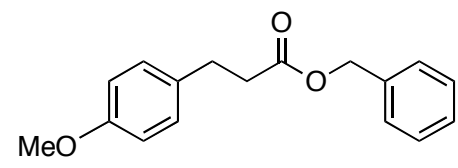

Benzyl 3-(4-methoxyphenyl)propanoate (8): Purified with $10 \%$ $\mathrm{Et}_{2} \mathrm{O} /$ hexanes yielding $127 \mathrm{mg}(76 \%)$ of $\mathbf{8}$ as a light yellow oil. $\mathrm{R}_{f}=$ 0.6 (1:4 Et 2 O/hexanes); IR (film) 3448, 3033, 2951, 2835, 1734, $1247 \mathrm{~cm}^{-1} ;{ }^{1} \mathrm{H}$ NMR $\left(500 \mathrm{MHz}, \mathrm{CDCl}_{3}\right) \delta$ 7.40-7.30 (m, 5H); 7.10

5. (a) Rivas, F. M.; Riaz, U.; Giessert, A.; Smulik, J. A.; Diver, S. T. Org. Lett. 2001, 3, 2673-2676. (b) Rivas, F. M.; Giessert, A. J.; Diver, S. T. J. Org. Chem. 2002, 67, 1708-1711. 
$(\mathrm{d}, J=7.94,2 \mathrm{H}) ; 6.82(\mathrm{~d}, J=7.63,2 \mathrm{H}) ; 5.11(\mathrm{~s}, 2 \mathrm{H}) ; 3.79(\mathrm{~s}, 3 \mathrm{H}) ; 2.92(\mathrm{t}, J=7.63,2 \mathrm{H}) ; 2.65(\mathrm{t}$, $J=7.63,1 \mathrm{H}) ;{ }^{13} \mathrm{C}$ NMR $\left(125 \mathrm{MHz}, \mathrm{CDCl}_{3}\right) \delta 173.3,158.5,136.4,132.9,129.7,129.0,128.7$, 128.6, 114.3, 66.7, 55.7, 36.7, 30.6; LRMS (APCI): Mass calculated for $\mathrm{C}_{17} \mathrm{H}_{18} \mathrm{O}_{3}[\mathrm{M}+\mathrm{H}]^{+}$271.3. Found 271.4.

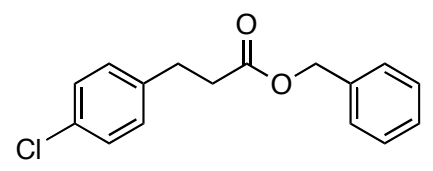

Benzyl 3-(4-chlorophenyl)propanoate (9): Purified with $10 \%$ $\mathrm{Et}_{2} \mathrm{O} /$ hexanes yielding $118 \mathrm{mg}(71 \%)$ of $\mathbf{9}$ as a yellow oil. $\mathrm{R}_{f}=0.75$ (1:4 Et $2 \mathrm{O} /$ hexanes); IR (film) 3452, 3033, 2949, $1735 \mathrm{~cm}^{-1} ;{ }^{1} \mathrm{H}$ NMR $\left(500 \mathrm{MHz}, \mathrm{CDCl}_{3}\right) \delta$ 7.37-7.22 (m, 7H); $7.11(\mathrm{~d}, J=8.2,2 \mathrm{H}) ; 5.10(\mathrm{~s}$, $2 \mathrm{H}) ; 2.94(\mathrm{t}, J=7.6,2 \mathrm{H}) ; 2.66(\mathrm{t}, J=7.6,2 \mathrm{H}) ;{ }^{13} \mathrm{C} \mathrm{NMR}\left(125 \mathrm{MHz}, \mathrm{CDCl}_{3}\right) \delta 172.9,139.3$, 136.2, 132.5, 130.2, 129.1, 128.8, 128.7, 66.8, 36.2, 30.7; LRMS (APCI): Mass calculated for $\mathrm{C}_{16} \mathrm{H}_{15} \mathrm{ClO}_{z}[\mathrm{M}+5 \mathrm{H}]^{+}$279.7. Found 279.5.

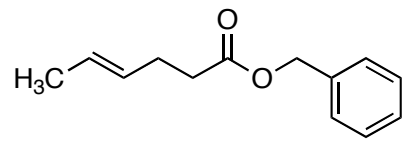

(E)-Benzyl hex-4-enoate (11): Purified with $10 \% \quad \mathrm{Et}_{2} \mathrm{O} / \mathrm{h}$ exanes yielding $129 \mathrm{mg}(70 \%)$ of $\mathbf{1 1}$ as a clear oil. $\mathrm{R}_{f}=0.77\left(1: 4 \mathrm{Et}_{2}\right.$ O/hexanes); IR (film) 3455, 3040, 2959, 2936, $1736 \mathrm{~cm}^{-1} ;{ }^{1} \mathrm{H}$ NMR $\left(500 \mathrm{MHz}, \mathrm{CDCl}_{3}\right) \delta$ 7.40-7.32 (m, 5H); 5.50-5.39 (m, 2H); $5.12(\mathrm{~s}, 2 \mathrm{H}) ; 2.41(\mathrm{~m}, 2 \mathrm{H}) ; 2.33(\mathrm{t}, J$ $=6.4,2 \mathrm{H}) ; 1.62(\mathrm{t}, J=5.8,3 \mathrm{H}) ;{ }^{13} \mathrm{C}$ NMR $\left(125 \mathrm{MHz}, \mathrm{CDCl}_{3}\right) \delta 173.3,136.3,129.3,128.7$, $128.4,126.5,66.3,34.5,28.1,22.6,18.1$; LRMS (APCI): Mass calculated for $\mathrm{C}_{13} \mathrm{H}_{16} \mathrm{O}_{2}[\mathrm{M}+\mathrm{H}]^{+}$ 205.2. Found 205.2.

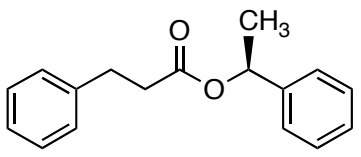

(S)-1-Phenylethyl 3-phenylpropanoate (6): Purified with 10\% $\mathrm{Et}_{2} \mathrm{O} /$ hexanes yielding $155 \mathrm{mg}(77 \%)$ of $\mathbf{6}$ as a yellow oil. $\mathrm{R}_{f}=0.70(1: 4$ $\mathrm{Et}_{2} \mathrm{O} / \mathrm{hexanes}$ ); IR (film) 3446, 3030, 2981, 2868, 1732, $1248 \mathrm{~cm}^{-1} ;{ }^{1} \mathrm{H}$ NMR $\left(500 \mathrm{MHz}, \mathrm{CDCl}_{3}\right) \delta$ 7.36-7.17 (m, 10H); $5.89(\mathrm{q}, J=6.7,1 \mathrm{H})$; $2.96(\mathrm{t}, J=7.3,2 \mathrm{H}) ; 2.66(\mathrm{t}, J=5.7,2 \mathrm{H}) ; 1.51(\mathrm{~d}, J=6.7,3 \mathrm{H}) ;{ }^{13} \mathrm{C} \mathrm{NMR}\left(125 \mathrm{MHz}, \mathrm{CDCl}_{3}\right) \delta$ $172.4,141.8,140.7,129.6,128.8,128.7,128.5,128.0,126.4,126.3,36.3,31.1,22.4$; LRMS (ACPI): Mass calculated for $\mathrm{C}_{17} \mathrm{H}_{18} \mathrm{O}_{2}[\mathrm{M}+\mathrm{H}]^{+}$255.3. Found 255.4.

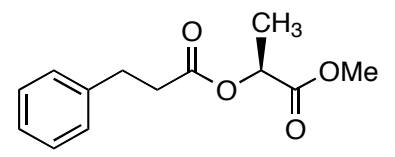

Methyl ester (7): Purified with $10 \% \mathrm{Et}_{2} \mathrm{O} /$ hexanes yielding $114 \mathrm{mg}$ (61\%) of 7 as a light yellow oil. $\mathrm{R}_{f}=0.54\left(1: 4 \mathrm{Et}_{2} \mathrm{O} /\right.$ hexanes); IR (film) $3463,3029,2994,2951,1744,1215 \mathrm{~cm}^{-1} ;{ }^{1} \mathrm{H}$ NMR $\left(500 \mathrm{MHz}, \mathrm{CDCl}_{3}\right) \delta$ 7.31-7.20 (m, 5H); $5.10(\mathrm{q}, J=7.0,1 \mathrm{H}) ; 3.74(\mathrm{~s}, 3 \mathrm{H}) ; 2.98(\mathrm{t}, J=7.9$, 2H); 2.75-2.70 (m, 2H); $1.47(\mathrm{~d}, J=7.3,3 \mathrm{H}) ;{ }^{13} \mathrm{C} \mathrm{NMR}\left(125 \mathrm{MHz}, \mathrm{CDCl}_{3}\right) \delta 172.5,171.5$, 140.5, 128.7, 128.5, 126.5, 68.7, 52.5, 35.7, 30.9, 17.1; LRMS (APCI): Mass calculated for $\mathrm{C}_{13} \mathrm{H}_{16} \mathrm{O}_{4}[\mathrm{M}+\mathrm{H}]^{+}$237.1. Found 237.1. 


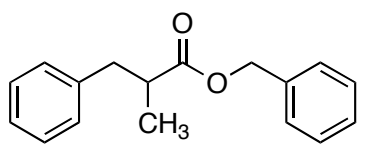

Benzyl 2-benzylpropanoate (12): Purified with $10 \% \mathrm{Et}_{2} \mathrm{O} /$ hexanes yielding $150 \mathrm{mg}(82 \%)$ of $\mathbf{1 2}$ as light $\tan$ oil. $\mathrm{R}_{f}=0.71(1: 4$ EtOAc/hexanes); IR (film) 3448, 3064, 3030, 3974, 2936, 1735, 1245 $\mathrm{cm}^{-1} ;{ }^{1} \mathrm{H}$ NMR $\left(500 \mathrm{MHz}, \mathrm{CDCl}_{3}\right) \delta$ 7.35-7.14 (m, 10H); $5.07(\mathrm{~s}, 2 \mathrm{H})$; $3.04(\mathrm{dd}, J=13.3,7.0,1 \mathrm{H}) ; 2.80(\mathrm{q}, J=7.3,1 \mathrm{H}) ; 2.69(\mathrm{dd}, J=13.4,7.3,1 \mathrm{H}) ; 1.18(\mathrm{~d}, J=6.7$, $3 \mathrm{H}) ;{ }^{13} \mathrm{C}$ NMR $\left(125 \mathrm{MHz}, \mathrm{CDCl}_{3}\right) \delta 176.4,139.7,136.5,129.5,129.0,128.8,128.6,128.5$, 126.8, 66.6. 42.0, 40.2, 17.3; LRMS (APCI): Mass calculated for $\mathrm{C}_{17} \mathrm{H}_{18} \mathrm{O}_{2}[\mathrm{M}+\mathrm{H}]^{+}$255.3. Found 255.3.

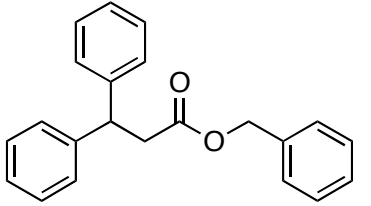

Benzyl 3,3-diphenylpropanoate (13): Purified with $10 \% \mathrm{Et}_{2} \mathrm{O} /$ hexanes yielding $113 \mathrm{mg}(82 \%)$ of $\mathbf{1 3}$ as a light yellow oil. $\mathrm{R}_{f}=0.70(1: 4$ $\mathrm{Et}_{2} \mathrm{O} /$ hexanes); IR (film) 3448, 3061, 3030, 2953, 2921, 1735, $1258 \mathrm{~cm}^{-1}$; ${ }^{1} \mathrm{H}$ NMR $\left(500 \mathrm{MHz}, \mathrm{CDCl}_{3}\right) \delta$ 7.38-7.14 (m, 15H); $5.02(\mathrm{~s}, 2 \mathrm{H}) ; 4.56(\mathrm{t}, J$ $=8.2,1 \mathrm{H}) ; 3.12(\mathrm{~d}, J=7.9,2 \mathrm{H}) ;{ }^{13} \mathrm{C} \mathrm{NMR}\left(125 \mathrm{MHz}, \mathrm{CDCl}_{3}\right) \delta 172.1,143.8,136.2,129.6$, $129.0,128.9,128.8,128.7,128.6,128.5,128.4,128.1,127.0,66.8,47.5,41.3$.

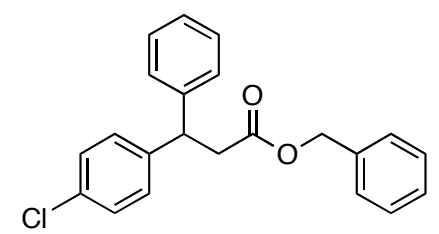

Benzyl 3-(4-chlorophenyl)-3-phenylpropanoate (14): Purified with $60 \% \mathrm{CH}_{2} \mathrm{Cl}_{2} /$ hexanes yielding $94 \mathrm{mg}(86 \%)$ of 14 as a light yellow oil. $\mathrm{R}_{f}=0.85$ (5:1 $\mathrm{CH}_{2} \mathrm{Cl}_{2} /$ hexanes); IR (film) 3449, 3030, 2955, $1735,1254 \mathrm{~cm}^{-1} ;{ }^{1} \mathrm{H} \mathrm{NMR}\left(500 \mathrm{MHz}, \mathrm{CDCl}_{3}\right) \delta$ 7.31-7.14 (m, 14H); $5.02(\mathrm{~s}, 2 \mathrm{H}) ; 4.54(\mathrm{t}, J=7.9,1 \mathrm{H}) ; 3.08(\mathrm{~d}, J=7.7,2 \mathrm{H}) ;{ }^{13} \mathrm{C}$ NMR $\left(125 \mathrm{MHz}, \mathrm{CDCl}_{3}\right) \delta 171.9,143.3,142.2,136.1,132.8,129.5,129.2,129.0,128.7 .128 .6,128.0$, 127.2, 114.6, 66.9, 46.9, 41.2.

\section{General Procedure for the Kinetic Resolution of Vinylogous Aldehydes:}

A screw-capped tube was charged with the chiral benzimidazolium salt $\mathbf{D}(42 \mathrm{mg}, 0.09 \mathrm{mmol})$ in a nitrogen-filled dry box. The tube was removed from the box and placed under a positive pressure of nitrogen. The tube was charged with toluene $(0.6 \mathrm{~mL}), \mathrm{DBU}(14 \mu \mathrm{L}, 0.09 \mathrm{mmol})$ and lastly, distilled cinnamaldehyde $(247 \mathrm{mg}, 1.87 \mathrm{mmol})$. The reaction mixture was allowed to stir for 5 minutes after which 2,6-dimethylphenol $(76 \mathrm{mg}, 0.62 \mathrm{mmol})$ in toluene $(0.6 \mathrm{ml})$ was added via syringe followed by the addition of 1-phenylethanol $(429 \mathrm{mg}, 0.62 \mathrm{mmol})$ and dodecane (106 $\mathrm{mg}, 0.62 \mathrm{mmol})$ that served as an internal standard for $\mathrm{GC}$ analysis. The reaction was heated at $80^{\circ} \mathrm{C} 6$ days and monitored by standard analytical techniques (GC and HPLC) for $\%$ conversion and enantiomeric excess values. Aliquots of the reaction mixture $(50 \mu \mathrm{L})$ were collected after $24 \mathrm{~h}, 48 \mathrm{~h}, 72 \mathrm{~h}, 96 \mathrm{~h}, 120 \mathrm{~h}$, and $144 \mathrm{~h}$. Each aliquot was filtered through a small silica gel plug (1:1 EtOAc/hexanes) and analyzed. ${ }^{6}$

\footnotetext{
${ }^{6}$ Percent conversions were measured by GC integration of the sec-phenethyl alcohol and the dodecane peaks.
} 
Table SI 1. Methods utilized for the determination of enantiomeric exdess.

\begin{tabular}{lccccc}
\hline Entry & Catalyst & ee Assay & Condition & $\begin{array}{c}\text { Retention Time } \\
\text { of (S) isomer (min) }\end{array}$ & $\begin{array}{c}\text { Retention Time } \\
\text { of (S) isomer (min) }\end{array}$ \\
\hline 1 & D & $\begin{array}{c}\text { HPLC } \\
\text { Chiralcel OD-H }\end{array}$ & $\begin{array}{c}0.5 \% \text { IPA/hexane } \\
0.75 \mathrm{~mL} / \mathrm{min}\end{array}$ & 9.76 & 11.14 \\
\hline
\end{tabular}

Table SI 2. Methods utilized for the determination of $\%$ conversion.

\begin{tabular}{lcccc}
\hline Entry & Catalyst & GC Conditions & $\begin{array}{c}\text { Retention Time of } \\
\text { sec-phenethyl alcohol (min) }\end{array}$ & $\begin{array}{c}\text { Retention Time of } \\
\text { dodecane (min) }\end{array}$ \\
\hline 1 & $\mathrm{D}$ & $\begin{array}{c}70^{\circ} \mathrm{C}, 1 \mathrm{~min} ; \\
35^{\circ} \mathrm{C} / \mathrm{min} \text { to } 285^{\circ} \mathrm{C} ; \\
285^{\circ} \mathrm{C}, 3 \mathrm{~min}\end{array}$ & 4.44 & 5.39 \\
& & & \\
\hline
\end{tabular}

Table SI 3. Selected Experimental Data for the Deternination of Conversion, Enantiomeric Excess, and Selectivity (s).

\begin{tabular}{lccccc}
\hline Entry & Catalyst & time (h) & \% conversion & Measured \% ee & s \\
\hline \multirow{2}{*}{1} & 54 & 32.4 & 34.2 & 8.25 \\
& D & 76 & 33.2 & 32.3 & 7.08 \\
& 100 & 40.7 & 25.3 & 2.93 \\
& 124 & 41.0 & 24.2 & 2.66 \\
& 150 & 28.9 & 3.15 \\
\hline
\end{tabular}




\section{Selected NMR Spectra}

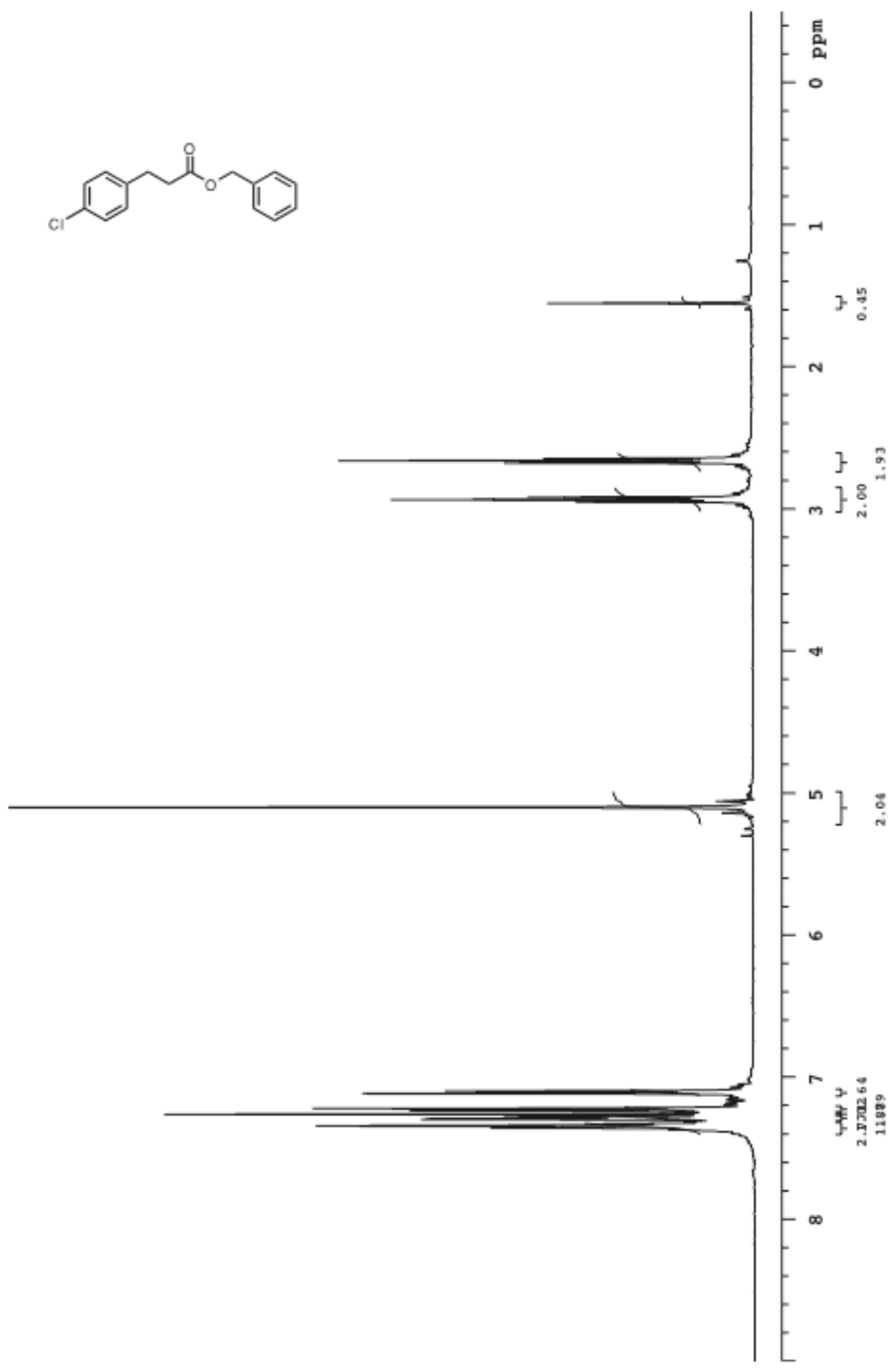




$$
\exists
$$




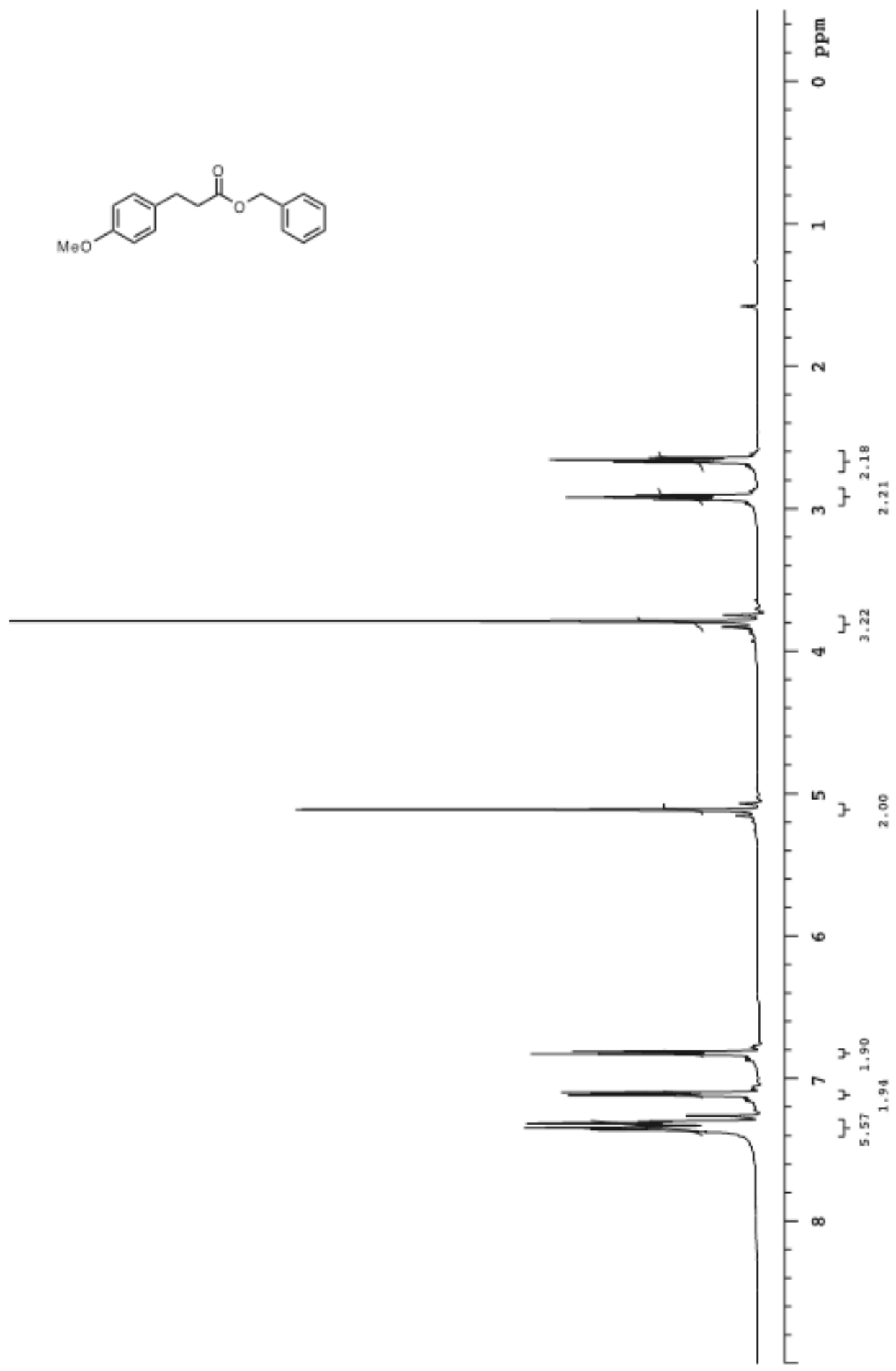


Org. Lett. Supporting Information

Page S9

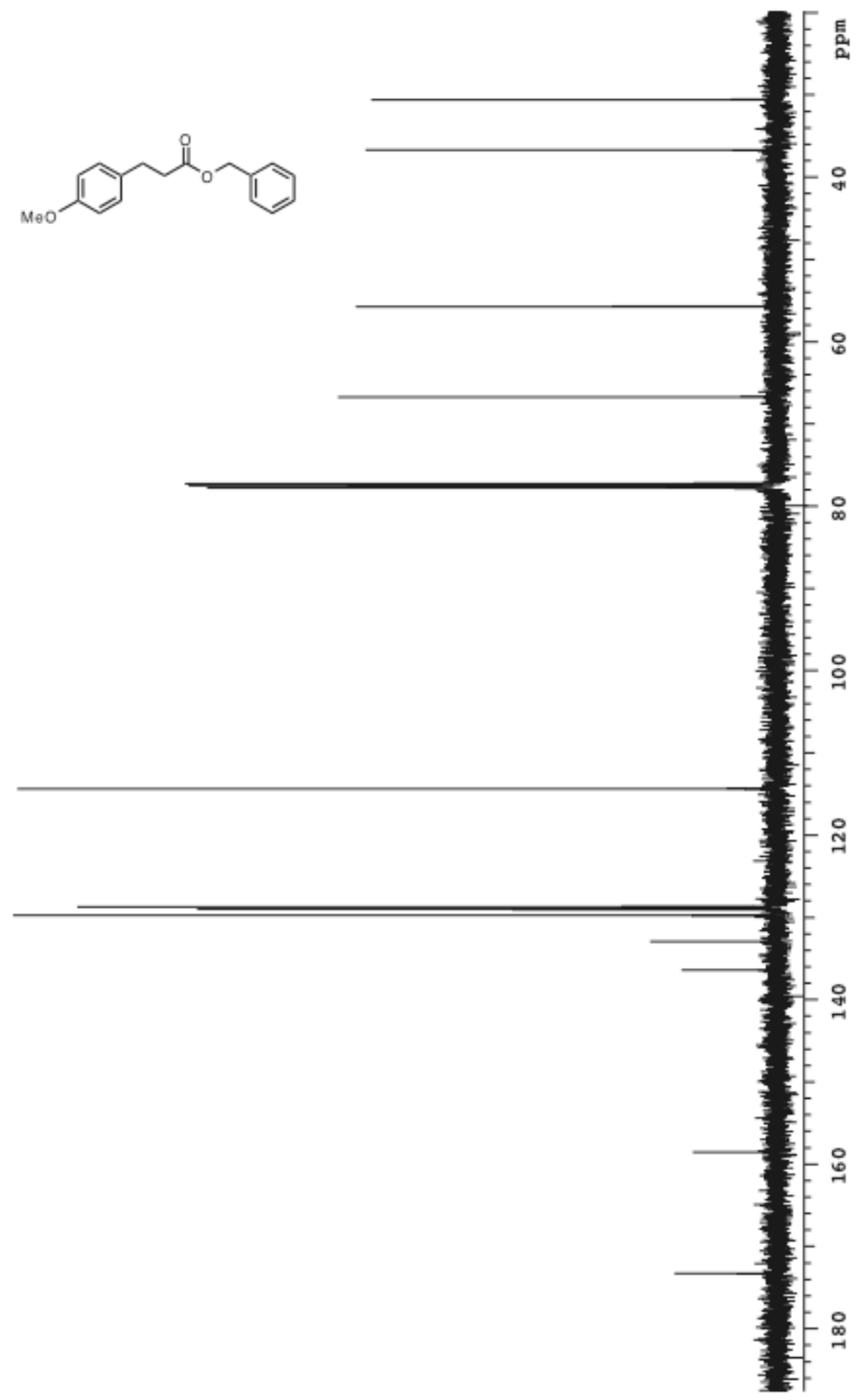




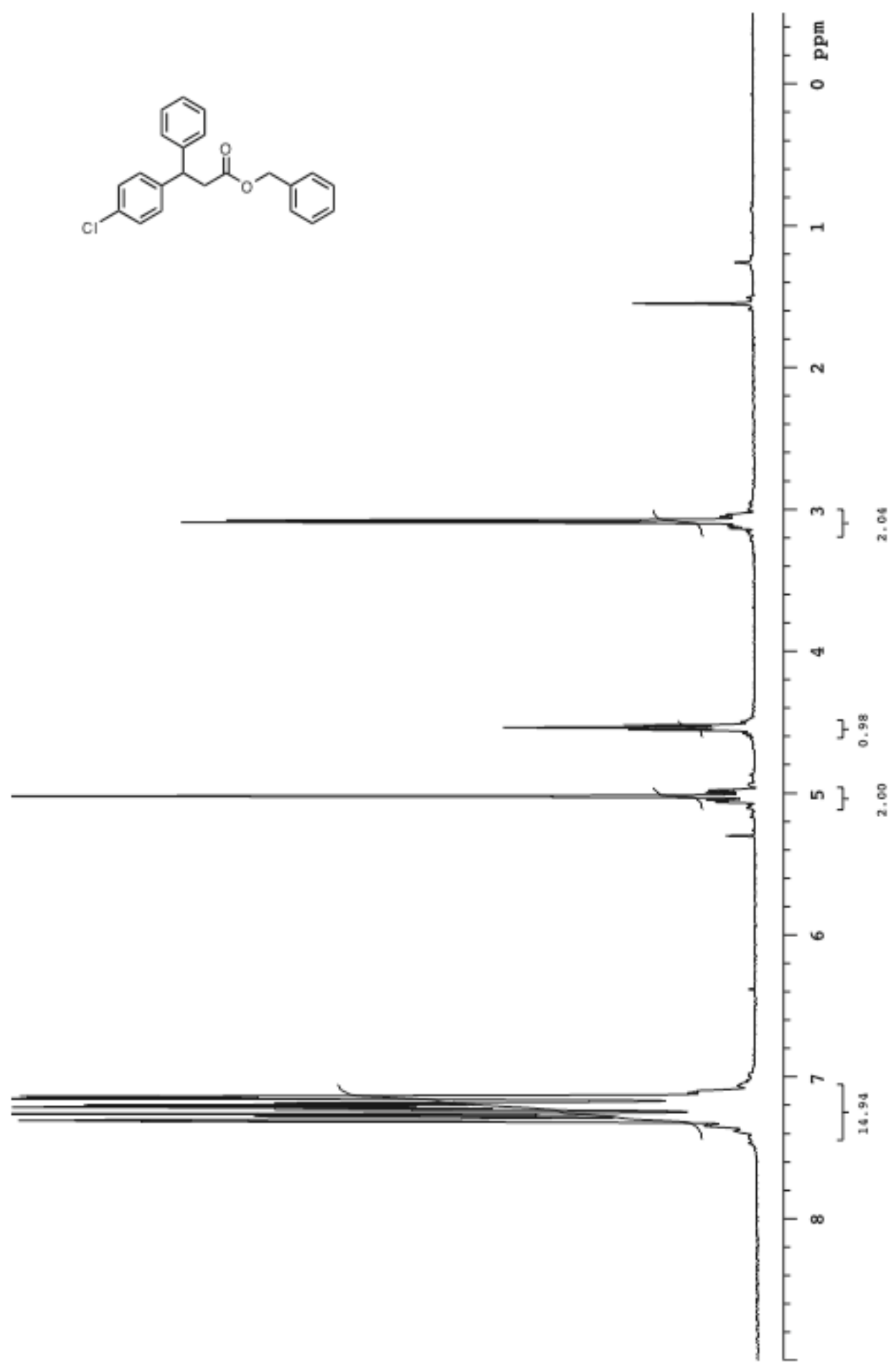




$$
1
$$



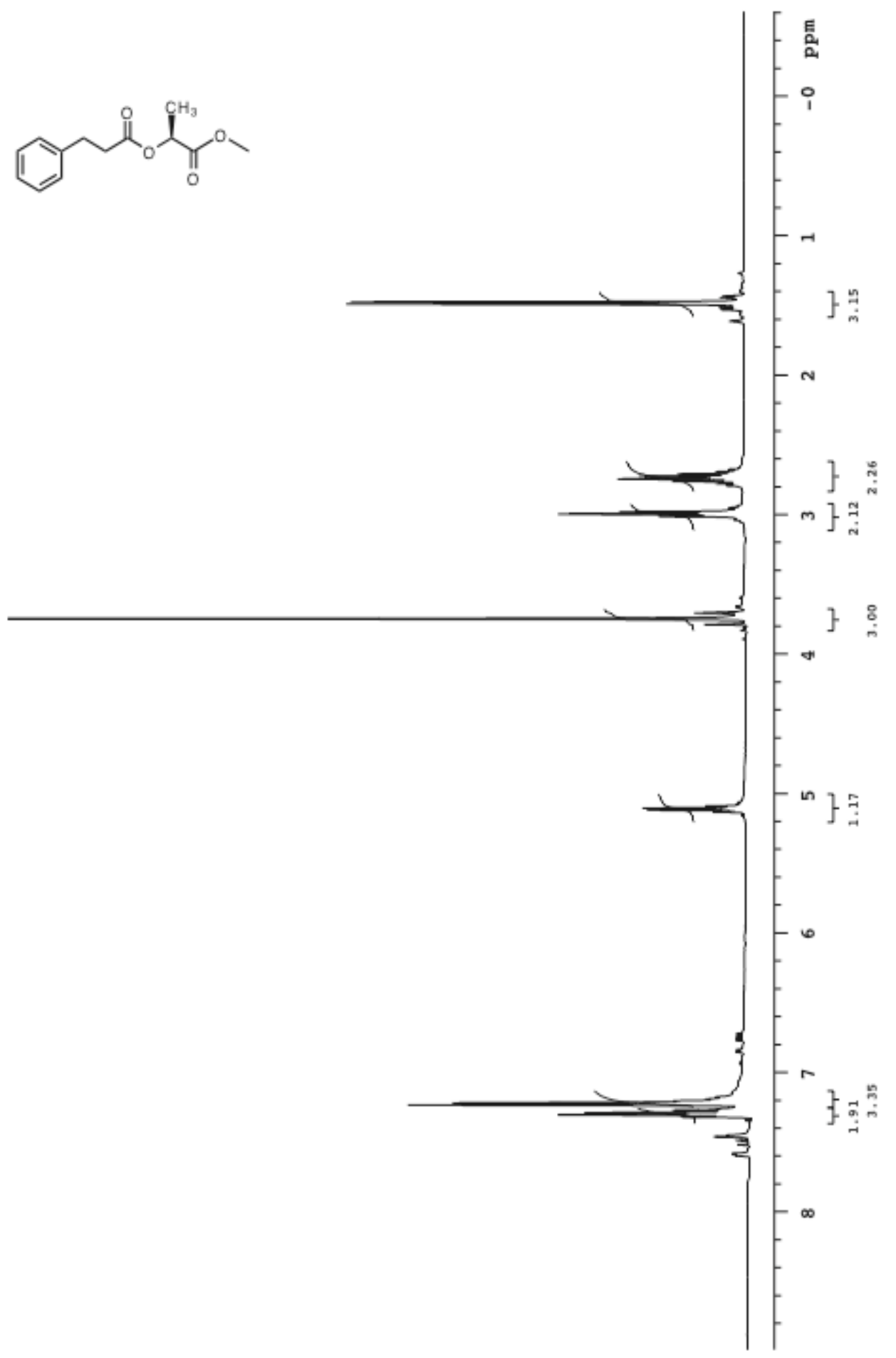


$$
1
$$


Org. Lett. Supporting Information

Page S14

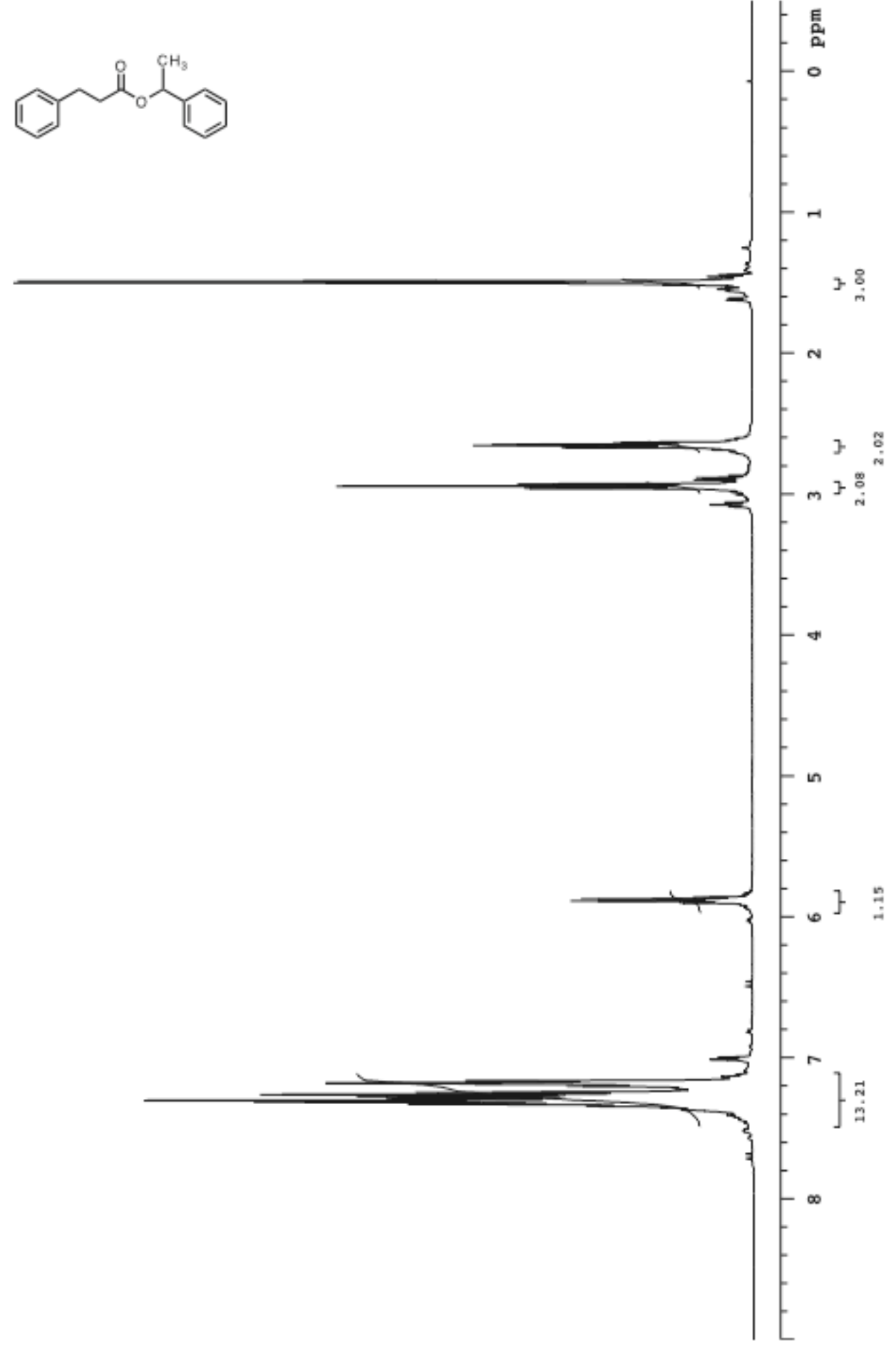




$$
\exists
$$


Org. Lett. Supporting Information

Page S16
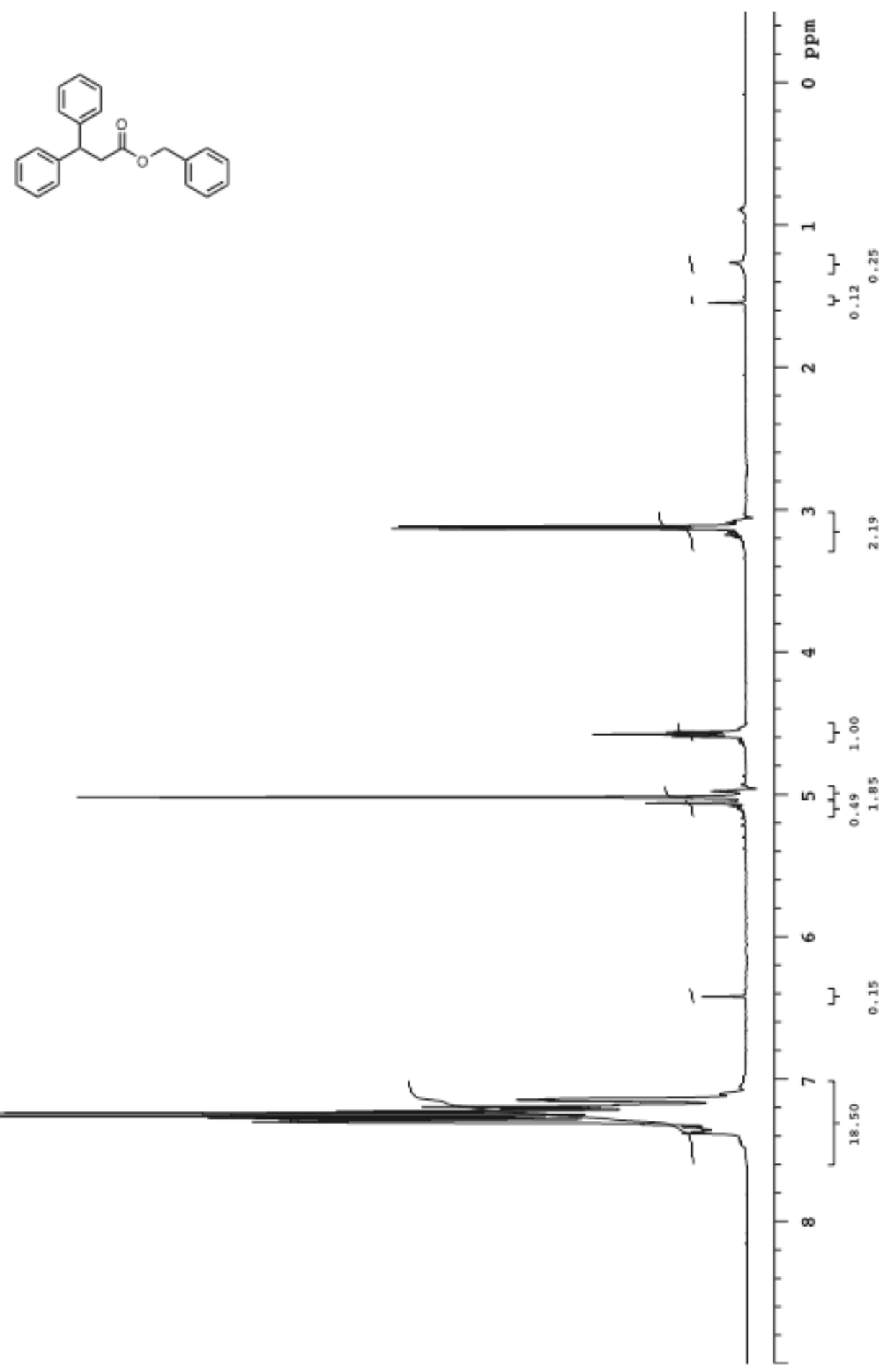
Org. Lett. Supporting Information

Page S17

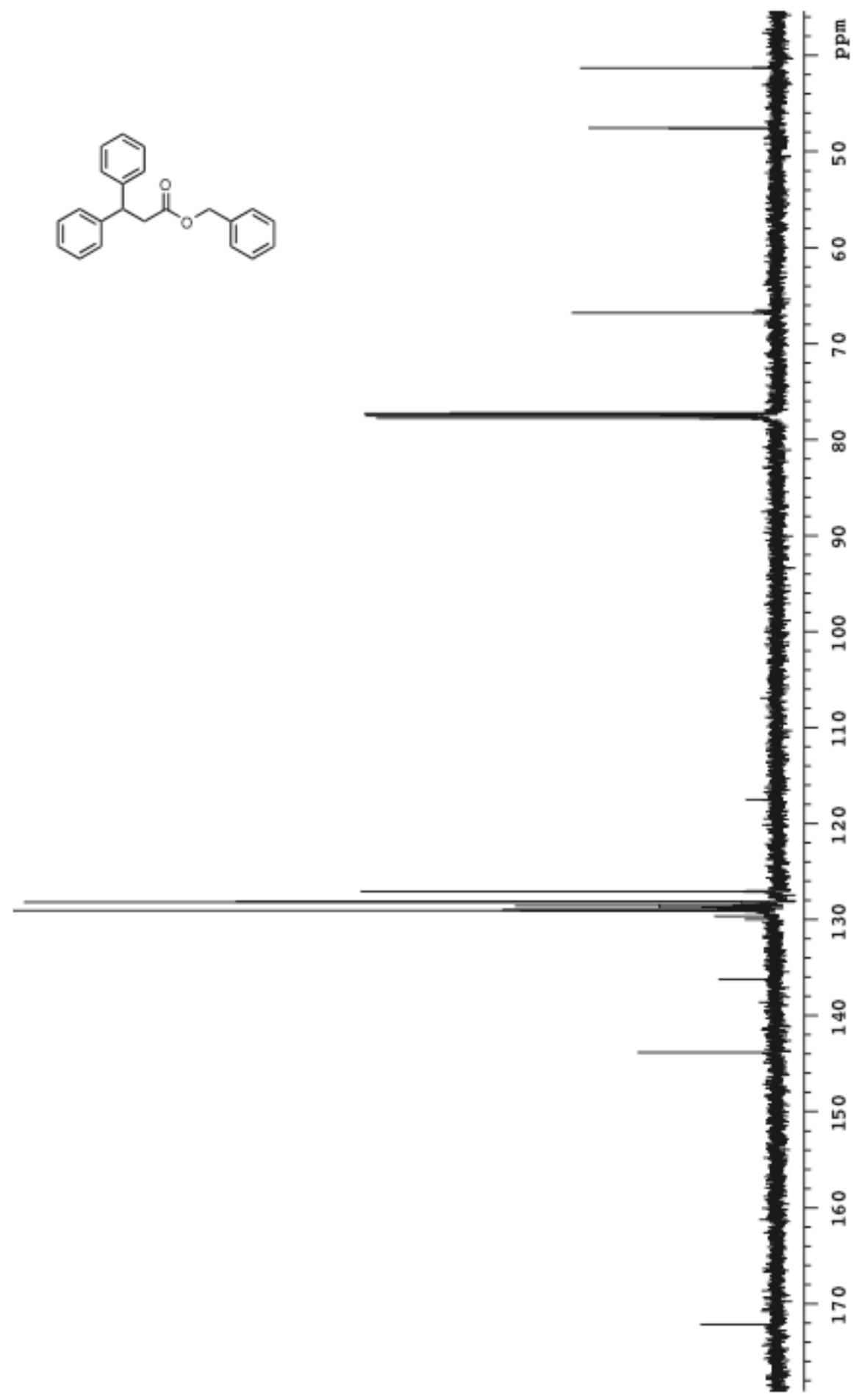




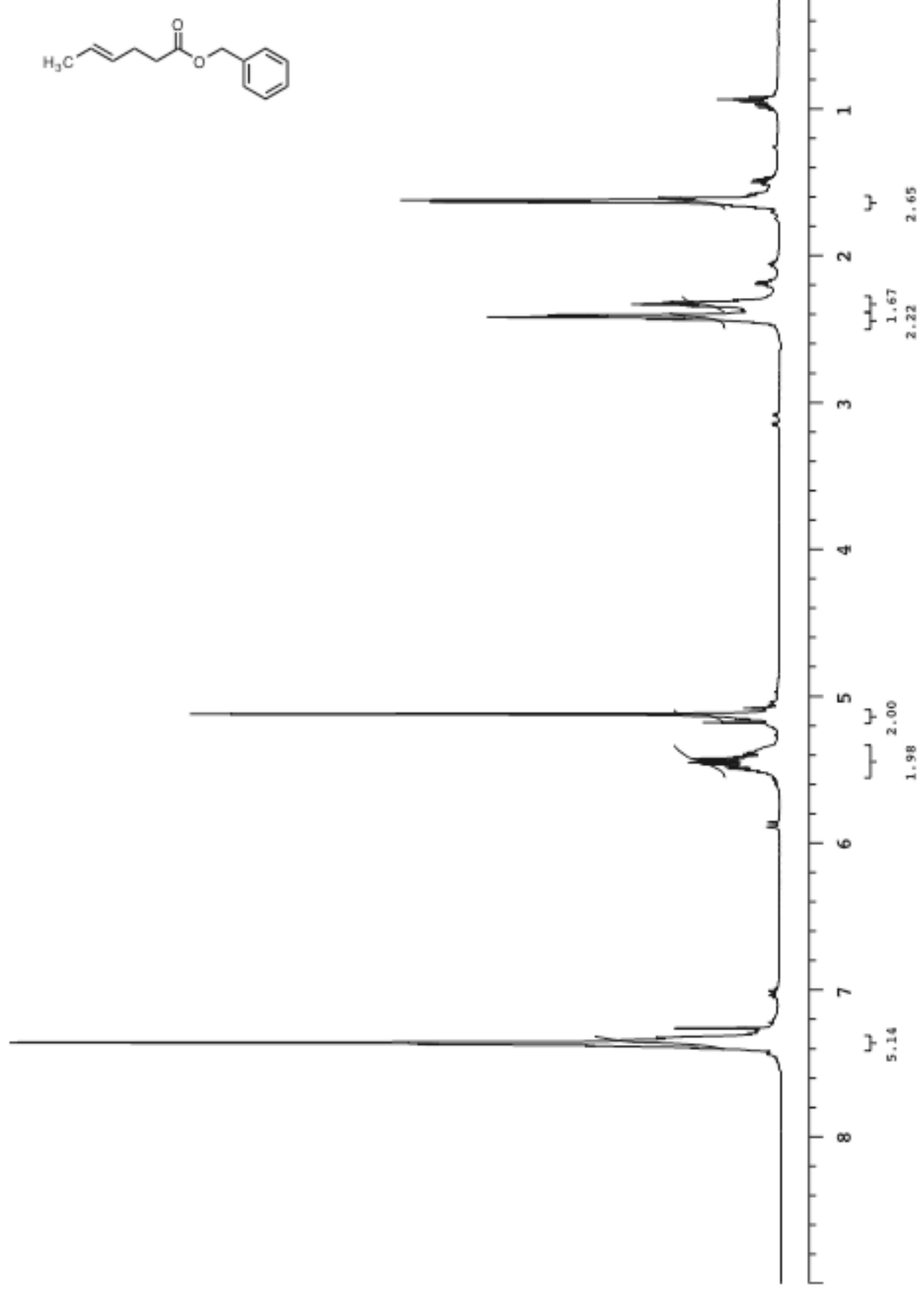




$$
\exists
$$




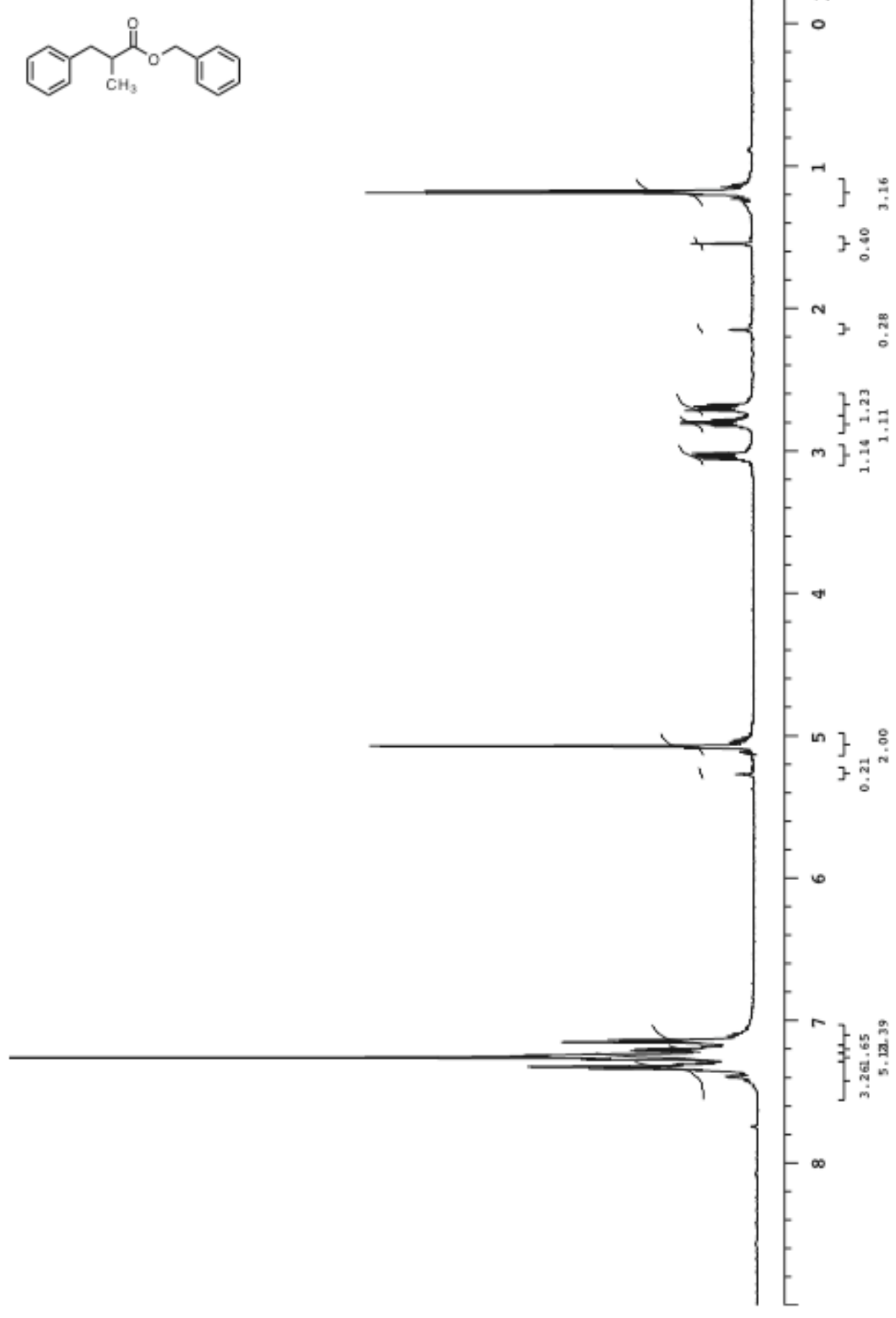




$$
\neq
$$

